\title{
Erratum zu: Twittern als \#Alltagspraxis des Kunstpublikums
}

\section{Marcus Müller · Jörn Stegmeier}

Online publiziert: 21. September 2017

(C) Springer-Verlag GmbH Deutschland 2017

\section{Erratum zu:}

Z Literaturwiss Linguistik (2016)

https://doi.org/10.1007/s41244-016-0036-0

Bei der Veröffentlichung dieses Beitrags enthielten die Tabellen 1 und 2 auf S. 505 teilweise fehlerhafte Werte. Die korrigierten Angaben finden Sie hier:

Tab. 1 Zusammensetzung des Korpus in absoluten Zahlen

\begin{tabular}{lll}
\hline & Englisch & Deutsch \\
Gesammelte Nachrichten & 10.494 & 437 \\
Tweets & 2922 & 193 \\
davon wurden retweetet & 601 & 40 \\
Anzahl Retweets & 7572 & 244 \\
\hline
\end{tabular}

Die Online-Version des Originalartikels ist unter https://doi.org/10.1007/s41244-016-0036-0 zu finden.

M. Müller $(\bowtie)$

TU Darmstadt, 64293 Darmstadt, Deutschland

E-Mail: mueller@linglit.tu-darmstadt.de

\section{J. Stegmeier}

Universität Heidelberg, 69117 Heidelberg, Deutschland

E-Mail: joern.stegmeier@gs.uni-heidelberg.de 
Tab. 2 Zusammensetzung des Korpus in relativen Zahlen

\begin{tabular}{lll}
\hline & Englisch \% & Deutsch \% \\
Tweets (Bezug: Gesammelte Nachrichten) & 27,84 & 44,16 \\
Retweetete Tweets (Bezug: Tweets) & 20,57 & 20,73 \\
Anzahl Retweets (Bezug: Gesammelte Nachrichten) & 72,16 & 55,84 \\
\hline
\end{tabular}

Zudem wurden versehentlich die Abbildungen 3 und 4 vertauscht, sodass die jeweiligen Bildunterschriften falsch zugeordnet sind. Die Korrekturen im Folgenden beziehen sich auf die tatsächlich abgedruckte Reihenfolge der Abbildungen: Auf S. 515, Absatz 3, muss es im Klammerausdruck „Abb. 4“ (statt „Abb. 2“) heißen. Auf S. 518, Absatz 1, muss es ,wie sie in Abb. 4 dokumentiert ist“ (statt ,wie sie in Abb. 3 dokumentiert ist"“) heißen.

Die Autoren und der Verlag bitten, diese Versehen zu entschuldigen. 